\title{
Vienne à l'écoute de Prague. La vie musicale pragoise selon la revue Musikblätter des Anbruch (1919-1937)
}

Jean-François Candoni, Wien hört Prag zu. Prager Musikleben nach der Zeitschrift Musikblätter des Anbruch (1919-1937)

Jean-François Candoni, Vienna listens to Prague. Prague musical life according to the magazine Musikblätter des Anbruch (1919-1937)

\section{Jean-François Candoni}

\section{(2) OpenEdition}

\section{Journals}

Édition électronique

URL : https://journals.openedition.org/austriaca/697

DOI : 10.4000/austriaca.697

ISSN : 2729-0603

Éditeur

Presses universitaires de Rouen et du Havre

Édition imprimée

Date de publication : 1 décembre 2019

Pagination : 63-76

ISBN : 979-10-240-1454-8

ISSN : 0396-4590

\section{Référence électronique}

Jean-François Candoni, « Vienne à l'écoute de Prague. La vie musicale pragoise selon la revue

Musikblätter des Anbruch (1919-1937) », Austriaca [En ligne], 88-89 | 2019, mis en ligne le 31 décembre 2020, consulté le 24 octobre 2022. URL : http://journals.openedition.org/austriaca/697 ; DOI : https:// doi.org/10.4000/austriaca.697 


\section{Vienne à l'écoute de Prague La vie musicale pragoise selon la revue Musikblätter des Anbruch (1919-1937)}

Fondée en 1919, la revue Musikblätter des Anbruch. Halbmonatsschrift für moderne Musik ${ }^{1}$ constitue une exception dans le paysage de la presse viennoise, tant en raison de son objet, la musique ${ }^{2}$, que de ses choix éditoriaux, qui accordent une attention toute particulière à la nouvelle musique et, plus généralement, à la vie musicale contemporaine au moment où le développement de l'industrie musicale, le mouvement de muséification des goûts musicaux et la patrimonialisation du répertoire semblent irréversibles. En proposant principalement des articles de fond à une époque où la critique de l'interprétation prend résolument le pas sur la critique de l'œuvre 3 , la revue se démarque résolument de ce que la presse quotidienne généraliste offre à ses lecteurs (c'est-à-dire essentiellement des comptes rendus de concerts) et elle est pratiquement sans concurrence dans le monde germanique, à l'exception notable de Melos, publiée à la même époque à Leipzig (1920-1934). Il y a d'ailleurs, dans les années 1920-1930, une forte rivalité entre presse spécialisée et presse quotidienne - jugée trop conservatrice - qui est montée en épingle de façon assez récurrente aussi bien dans Musikblätter des Anbruch que dans Melos.

Porte-étendard de la modernité musicale, la revue viennoise est publiée dans un premier temps par les éditions Universal, puis, à partir de 1935, par le Vorwärts-Verlag. Elle réunit quelques-uns des plus prestigieux musicographes de l'époque : des élèves de Schönberg tels que

1. À partir de 1929, la revue s'appelle simplement Anbruch.

2. Il existe dans la période de l'entre-deux-guerres deux autres revues musicales viennoises : Der Merker, qui interrompt sa publication en 1922, et Eine Wiener Musikzeitschrift (1932-1937), laquelle se désintéresse totalement de la vie musicale tchèque.

3. Voir, à ce sujet, Carl Dahlhaus, «Vom Elend der Musikkritik» (Melos, 1957), dans Carl Dahlhaus, Gesammelte Schriften 2, Laaber, Laaber, 2001, p. 83-84 ; ainsi que : Joachim Kaiser, "Glanz und Elend der Musikkritik», Schweizer Monatshefte. Zeitschrift für Politik, Wirtschaft, Kultur, vol. 68, nº 5, 1988, p. 425-440. 
Paul Stefan (qui devient rapidement le principal contributeur) et Egon Wellesz, des critiques renommés tels que Paul Bekker, Hans Heinz Stuckenschmidt et Oskar Bie, l'écrivain pragois Max Brod, ou des intellectuels comme Ernst Bloch. Theodor W. Adorno y publie quelques-uns de ses essais les plus importants, et la revue offre une tribune à des compositeurs de tout premier plan tels qu'Alban Berg, Arnold Schönberg, Béla Bartók, Alois Hába, Erwin Schulhoff ou Ernst Křenek.

Elle est en outre la seule revue viennoise à accorder une place de choix à la vie musicale hors d'Autriche et en particulier, pour le sujet qui nous intéresse ici, à la vie musicale pragoise (il y est largement question aussi de la Russie, de l'Italie et de la France) : il s'agit de réaffirmer par ce biais la vocation culturelle internationale de l'ancienne capitale des Habsbourg. Mais la volonté de dépasser la perspective traditionnelle, héritée du XIX ${ }^{e}$ siècle, d'analyse de la musique en termes d'écoles nationales est affirmée avec force dès l'article inaugural de Guido Adler :

Quel lieu serait plus approprié pour rétablir les relations internationales dans le domaine de la musique que ce sol viennois, consacré par le classicisme, et dont la vocation historique et la mission pour l'avenir est de médiatiser dans toutes les phases de leur évolution le nord et le sud, l'est et l'ouest ${ }^{4}$ ?

Les nombreux articles, ainsi que plusieurs dossiers ou numéros spéciaux, consacrés à la musique à Prague jettent un éclairage intéressant sur un phénomène totalement passé sous silence dans la presse quotidienne, qui adopte un point de vue exclusivement viennois : le caractère fortement dissymétrique de la relation entre Prague et Vienne. Ce phénomène est à l'évidence le résultat des vicissitudes de l'histoire : tandis que, dans la capitale tchèque, la musique allemande fait partie intégrante du paysage musical (toutes les institutions sans exception accordent une place importante aux compositeurs allemands dans leur programmation régulière), la musique tchèque est beaucoup moins présente à Vienne, où elle est accueillie avec une bienveillance parfois légèrement teintée de condescendance, et où les opéras de Bedřich Smetana ou de Leoš Janáček sont toujours donnés dans des traductions allemandes par les troupes d'opéra autrichiennes. À l'Opéra de Vienne en particulier, si

4. Guido Adler, «Zum Geleite», Musikblätter des Anbruch, novembre 1919, p. $1:$ «Welcher Ort wäre besser geeignet, die internationalen Beziehungen auf dem Gebiete der Musik wiederherzustellen, als der klassisch geweihte Boden Wiens mit der historischen und zukünftigen Mission der Ausgleichung aller Entwicklungsmomente von Nord und Süd, von Ost und West!» 
l'on excepte La Fiancée vendue (Die verkaufte Braut / Prodaná nevěsta), imposée par Gustav Mahler dès 1894, et des reprises sporadiques de Dalibor de Smetana, les grandes œuvres du répertoire tchèque (Antonín Dvořák et Leoš Janáček) sont quasiment exclues du répertoire courant, et il faut attendre la tournée d'une troupe venue d'Olomouc en juin 1924 pour que l'on assiste à un véritable cycle d'opéras tchèques, qui comprend notamment, outre Dalibor, Les Deux Veuves (Dvě vdovy), Le Secret (Tajemstvi) et Le Mur du diable (Čertova stěna) de Smetana, Catherine et Diable (Čert a Káča) et Rusalka de Dvořák.

Les Musikblätter des Anbruch offrent une ouverture sur un univers musical mal connu du lectorat autrichien et allemand. Certes, les articles traitant de la musique contemporaine tchèque sont en majorité rédigés par des correspondants pragois tchècophones (ils sont dans ce cas traduits du tchèque) ou, plus rarement, germanophones - c'est le cas notamment des textes consacrés à Leoš Janáček, dus pour la plupart à la plume de Max Brod. Cela s'explique sans doute en partie pour des raisons de compétence : la musique contemporaine tchèque étant très peu jouée à Vienne, il était difficile d'en rendre compte sans solliciter des musicographes pragois. Cela ne va pas sans des divergences d'approche entre, d'une part, les contributeurs pragois, qui se placent encore largement, que ce soit conscient ou non, dans une optique de musique nationale et, d'autre part, les contributeurs viennois (ou allemands), pour qui la question du nationalisme musical est sinon idéologiquement problématique, du moins obsolète : «L'histoire des arts nous enseigne que la distance historique efface les différences liées au climat ${ }^{5}$ », constate ainsi Hans Heinz Stuckenschmidt. C'est certainement dans cette optique que Paul Bekker reproche à Alois Hába, dans son article sur Smetana, d'ignorer l'influence de Liszt sur le compositeur de Ma Patrie (Má Vlast) et de sous-estimer ainsi sa dimension universelle $e^{6}$.

Si de nombreuses contributions s'appliquent à définir les spécificités d'une musique moderne tchèque, on ne trouve pas d'équivalent dans la revue quant à la définition d'une musique moderne autrichienne ou viennoise : il y est généralement simplement question de musique allemande, au sens large du terme. Les Musikblätter des Anbruch n'offrent

5. Hans-Heinz Stuckenschmidt, «Musikalische Autarkie», Anbruch, juin 1932, p. 106 : «Die Geschichte der Künste lehrt uns, daß historische Distanz die klimatischen Grenzen verwischt.»

6. Voir Paul Bekker, «Nationale und internationale Musik», Musikblätter des Anbruch, mai 1924, p. 175. 
en effet pas (du moins jusqu'en 1936) de tribune à ces musicographes nationalistes qui, à la même époque, cherchent à mettre en avant les caractéristiques purement autrichiennes de certains compositeurs, au premier chef Anton Bruckner?

\section{Répertoire tchèque et répertoire allemand à Prague : entre rivalité et médiation}

Les dossiers consacrés à Prague et à la musique tchèque en novembre 1919, en mai 1925 et en août 1935 dépeignent un univers artistique foisonnant, où se côtoient et s'interpénètrent deux cultures musicales distinctes, ce qui tient notamment au fait que la ville est non seulement «le centre de l'art tchèque, mais aussi celui de cet art des Allemands des Sudètes qu'on aurait tort de négliger ${ }^{8} »$. Cette richesse résulte en partie d'une forte émulation entre les trois grandes institutions qui dominent la vie musicale pragoise ${ }^{9}$ : l'Opéra national, l'Opéra allemand et la Philharmonie tchèque, auxquels on adjoindra le Conservatoire ${ }^{10}$.

Il n'y a pas de stricte répartition des répertoires entre les deux théâtres pragois - même si le premier, dirigé par Otakar Ostrčil (1920-1935), est nettement dominé par l'opéra tchèque et le second, qui connaît son apogée artistique sous la direction du Viennois Alexander von Zemlinsky (1911-1927), par l'opéra allemand et international. Et il arrive que les deux théâtres collaborent, comme lors de la fête de la musique de 1935. En revanche, plusieurs articles dénoncent la faiblesse des moyens financiers accordés au théâtre allemand, qui a dû quitter le Théâtre des États, rattaché au Théâtre national tchèque, pour s'installer à partir de 1921 dans le Neues Deutsches Theater, et est presque entièrement financé par des fonds privés, sans que les évidentes raisons politiques de cette inégalité de traitement soient clairement mises en avant par les contributeurs. La revue affirme toutefois que la créativité et l'innovation artistiques sont inversement proportionnelles au soutien financier accordé par les

7. Voir Erich Wolfgang Partsch, «Die Bruckner-Rezeption», dans Hans-Joachim Hinrichsen (dir.), Bruckner-Handbuch, Stuttgart, Metzler, 2010, p. 340 et suiv.

8. «Zu den Musikfesten im April», Anbruch, mars-avril 1936, p. 33 : «Prag, Mittelpunkt der tschechischen, aber auch einer nicht zu vernachlässigenden sudetendeutschen Kunst.»

9. Voir notamment : «Internationaler Kongress für Musikerziehung in Prag», Anbruch, mai 1936, p. 88.

10. Nous parlons ici des institutions (c'est-à-dire des troupes et des orchestres) et non des salles où elles se produisaient. 
autorités ${ }^{11}$. Les difficultés financières n'empêchent en effet pas le théâtre allemand - dont le public reste majoritairement germanophone - de mettre en place une programmation audacieuse, offrant la part belle à la musique contemporaine, en particulier grâce à l'engagement de son directeur musical : la saison 1922-1923, pour ne prendre qu'un exemple, ne propose pas moins de 53 opéras différents, avec une forte ouverture au répertoire international. Paradoxalement, la vie musicale allemande pragoise semble manquer de rayonnement en dehors de la Tchécoslovaquie, malgré le prestige dont jouit Zemlinsky à Prague, même auprès du public tchècophone ${ }^{12}$ - et les Musikblätter des Anbruch se fixent pour objectif d'y remédier. Les commentaires sur le théâtre national sont quant à eux plus mitigés, même si l'on reconnaît les efforts du compositeur et chef d'orchestre pragois Otakar Ostrčil pour valoriser la musique contemporaine ${ }^{13}$.

La troisième grande institution est la Philharmonie tchèque, dont le chef, Václav Talich (surnommé «le Furtwängler tchèque») a fait une phalange d'exception avec laquelle il cherche à se poser en médiateur entre la production musicale tchèque et la production austro-allemande - Zemlinsky est régulièrement invité à diriger l'orchestre. La carrière de Talich est placée sous le signe d'une double culture musicale : formé en partie à Berlin, où il fut l'assistant d'Arthur Nikisch, il dirige aussi bien le grand répertoire austro-allemand (Beethoven, Schubert, Brahms) que Smetana, Dvořák et la musique tchèque contemporaine, notamment les œuvres de son ami Josef Suk. Talich peut être considéré comme l'un des principaux artisans du rayonnement de la culture musicale pragoise en Europe, comme en témoignent les nombreuses tournées qu'il organisa avec son orchestre. Les comptes rendus des concerts qu'il donne à Vienne en décembre 1922 montrent cependant à quel point la musique tchèque est perçue en Autriche comme le produit d'un art

11. Voir Ernst Latzko, "Prager Musikwinter», Anbruch, novembre-décembre 1933, p. 151.

12. Voir notamment Felix Adler, "Zemlinsky», Musikblätter des Anbruch, mai 1923, p. 144 : «Davon weiß man, seit die Insel des Prager Deutschtums von der Außenwelt abgeschlossen ist, draußen sehr wenig."

13. Jan Löwenbach, "Tschechoslowakische Komponisten nach dem Kriege», Musikblätter des Anbruch, novembre 1922, p. 274 : «In Otakar Ostrčil (geb. 1879) hat zwar das Prager Nationaltheater einen neuen Opernchef von künstlerischem Rang und ernstem Wollen gefunden. Allein die locker gewordene Disziplin des Ensembles, die Unlust zum modernen Schaffen, die eingebildete Überlegenheit jedem Bühnenexperiment gegenüber hat seinen achtunggebietenden Bestrebungen den festen Boden bisher geradezu systematisch untergraben.» 
essentiellement national, sinon d'intérêt purement local. La critique rédigée par Ernst Décsey pour le Neues Wiener Tagblatt revient de manière insistante sur les arrière-plans nationaux, voire nationalistes, de la tournée de la Philharmonie tchèque, qu'il s'agisse du choix des œuvres exécutées - Ma Patrie de Smetana et Asrael de Suk («le politicien et l'artiste, étroitement liés, sont en quête d'expansion $\left.{ }^{14} »\right)$ - ou du style et du son de l'orchestre ( Il existe un son qui caractérise tout un peuple ; il est ici audible $\left.{ }^{15} »\right)$.

\section{Modernité et tradition : autour de la figure tutélaire de Bedřich Smetana}

Si aujourd'hui, c'est Dvořák qui est devenu la figure emblématique de la musique tchèque, le compositeur le plus cité, le plus commenté et le plus célébré dans l'entre-deux-guerres est sans conteste Bedřich Smetana, même dans une revue dédiée à la musique contemporaine - ce qui témoigne d'un fort attachement du monde musical tchèque à ses racines romantiques et nationales, mais révèle en même temps ses hésitations quant à la définition de la modernité. Que ce soit dans les Musikblätter des Anbruch ou bien dans la presse quotidienne viennoise, Smetana est systématiquement présenté comme le père de la musique tchèque. "Au commencement était Smetana ${ }^{16}$ ", répète le critique conservateur Julius Korngold dans la Neue Freie Presse à l'occasion du centenaire de sa naissance. En même temps, Korngold insiste sur l'importance qu'a eue le milieu culturel allemand pour la formation artistique du compositeur et affirme qu'il maîtrisait mal la langue tchèque. On retrouve dans un article du Neues Wiener Journal publié peu auparavant des remarques allant dans le même sens : «Son cœur politique battait au rythme tchèque. Son cœur musical éprouvait de l'enthousiasme et de l'amour pour la musique allemande ${ }^{17}$.» C'est un peu

14. Ernst Décsey, «Feuilleton. Musik», Neues Wiener Tagblatt, 11 décembre 1922, p. 3 : "Politiker und Künstler, aneinander gebunden, suchen die Expansion.»

15. Ibid. : «Es gibt den Eigenton eines ganzen Volkes; hier wird er hörbar.»

16. «Im Anfange war Smetana», in Julius Korngold, «Friedrich Smetana. Zur Hundertsten Wiederkehr seines Geburtstags», Neue Freie Presse, 2 mars 1924, p. 1 et suiv. Successeur d'Eduard Hanslick en tant que critique musical auprès de la Neue Freie Presse, père du compositeur Erich Wolfgang Korngold, Julius Korngold était l'un des critiques les plus réputés de l'époque.

17. Elsa Bienenfeld, «Smetana als Kritiker», Neues Wiener Journal, 24 février 1926, p. 12 : «Sein politisches Herz schlug in tschechischem Rhythmus. Sein musikalisches schwärmte und liebte die deutsche Musik.» 
comme si l'identité musicale tchèque, symbolisée par le compositeur de Libuše, n'avait pu se développer que sous l'impulsion de l'apport culturel du monde germanique, dont elle ne serait finalement, aux yeux de la presse conservatrice, qu'un sous-produit.

Dans les Musikblätter des Anbruch, c'est en premier lieu le rapport de Smetana à la modernité qui est interrogé : on souligne, à l'opposé des analyses de Julius Korngold (qui était un adversaire résolu de la Seconde École de Vienne et de l'atonalisme) et d'Ernst von Rychnowsky, qui situe Smetana dans le double héritage de Liszt et de Wagner ${ }^{18}$, la capacité du compositeur tchèque à dépasser le romantisme et la musique à programme chère au XIX ${ }^{\mathrm{e}}$ siècle : la volonté de rupture avec le wagnérisme et avec la Neudeutsche Schule est un présupposé essentiel des discours sur la modernité. On met par conséquent en avant la maîtrise formelle dont font preuve ses compositions : "Ce sont les qualités architectoniques de Smetana qui font de lui un compositeur vivant pour la musique tchèque moderne ${ }^{19}$.» Cette évolution dans l'appréciation de l'œuvre de Smetana présente un étonnant parallèle avec la réception de Bruckner en Autriche par la critique non conservatrice : d'abord vilipendé pour s'être fourvoyé dans la musique à programme, il a ensuite été élevé au rang de maître de la forme sonate, à la faveur notamment des travaux d'August $\mathrm{Halm}^{20}$.

Les défenseurs de la nouvelle musique mettent également en avant un autre aspect de la modernité de Smetana - qui n'est d'ailleurs pas tout à fait compatible avec le précédent : il serait le précurseur du réalisme musical et de Leoš Janáček, ce qui l'éloigne non seulement de la Neudeutsche Schule, mais également du travail formel caractéristique du classicisme viennois : "L’expressivité des opéras de Smetana (et en particulier sa rythmique) émanait de l'esprit de la déclamation de la langue tchèque », explique Alois Hába ${ }^{21}$. Ce dernier, dont le goût pour

18. Ernst von Rychnowsky, «Smetana, Liszt und Wagner», Anbruch, mars 1924, p. 84 et suiv. Il s'agit en fait de la reprise d'un extrait de la première biographie de Smetana publiée en langue allemande (Ernst von Rychnowsky, Smetana, Berlin, Deutsche Verlags-Anstalt, 1924). L'argumentaire déployé dans cet article détonne fortement par rapport aux grandes options de la revue.

19. Vladimir Helfert, "Zwei Gegenpole der tschechischen Musik: Smetana und Janáček», Anbruch, avril 1934, p. 64 : «Schon vermöge seiner architektonischen Tendenz ist Smetana für die moderne tschechische Musik lebendig."

20. August Halm, Die Symphonie Anton Bruckners, München, G. Müller, 1914.

21. Alois Hába, «Smetana und die neue Musik», Musikblätter des Anbruch, mars 1924, p. 82 : "die musikalische Ausdrucksweise der Opern Smetanas (insbesondere seine Rhythmik) entwuchs dem Geiste der Deklamation der tschechischen Sprache.» 
une modernité radicale lui a parfois valu d'être comparé à Schönberg ${ }^{22}$, ne nie certes pas l'importance de la logique musicale dans l'œuvre de Smetana, mais il l'interprète comme un moment essentiel du processus d'émancipation par rapport aux formes conventionnelles de la tradition classique et y voit une préfiguration des innovations formelles de la musique tchèque contemporaine. Les caractéristiques essentielles de cette rupture sont pour lui les suivantes :

élimination cohérente de toutes les caractéristiques stylistiques et architectoniques de l'époque classique (renoncement au principe de la répétition mélodique, de la transposition, du travail thématique, de la graduation séquencée des formes classiques réelles et de leurs composantes) - concentration sur une mélodie asymétrique et articulée librement qui progresse continuellement en séquences plus ou moins longues ${ }^{23} \ldots$

Dans l'article où il présente son propre opéra intitulé La Mère (Matka, 1931), Hába approfondit son propos à travers une analyse détaillée de son propre «style musical non thématique ${ }^{24}$ » et souligne à l'occasion à quel point cette œuvre jette un pont entre modernité et tradition (le principe de composition par quarts de ton est inspiré des chants populaires moraves).

Toute la difficulté consiste, pour les musicographes tchèques, à articuler un discours qui réaffirme sans cesse leur attachement à la culture populaire, liée au terroir, aux exigences d'une modernité qui s'est résolument détournée des revendications nationales et est en quête d'une universalité qui ne correspond pas nécessairement à une internationalisation. La recherche d'une médiation entre la prise en charge de la tradition musicale tchèque, incarnée par Smetana, et la valorisation de nouveaux moyens d'expression est une constante dans les discours sur la modernité tenus dans les Musikblätter des Anbruch. C'est sans doute l'écrivain pragois Jan Löwenbach (qui fut également le

22. Voir Oskar Kroll, «Anfänge der Vierteltonmusik», Anbruch, juin 1932, p. 109-110.

23. Alois Hába, "Smetana und die neue Musik», art. cité, p. 83-84 : "Eine konsequente Ausmerzung aller stilistischen und architektonischen Merkmale der klassischen Zeit (Verzichtleistung auf das Prinzip der melodischen Wiederholung, Transponierung, thematischen Arbeit, sequenzierenden Graduation, der realen klassischen Formen und ihrer Bestandteile) - Konzentration auf eine frei asymmetrisch gegliederte Melodik, welche unaufhörlich in größeren oder kleineren Abschnitten vorwärtsfließt.»

24. Alois Hába, «Meine Vierteltonoper: Die Mutter», Anbruch, mai 1931, p. 91 : «Der unthematische Musikstil.» 
traducteur de Rilke en tchèque) qui en résume le mieux les différents aspects : "exacerbation et complexification de moyens d'expression techniques, dissolution des fondements harmoniques, accentuation de la pure technicité et de l'élément subjectif», tout en "préservant la tradition ${ }^{25}$.» Et c'est en Janáček que Löwenbach pense reconnaître l'artiste possédant toutes ces qualités.

\section{La question de la musique nationale}

Dans la presse quotidienne viennoise, la musique tchèque est systématiquement associée à la question nationale. L'histoire des relations entre Vienne et la musique tchèque dans l'entre-deux-guerres s'ouvre sur un événement ayant une forte valeur symbolique, mais passé relativement inaperç $\mathrm{u}^{26}$, et ce malgré la publication de nombreuses recensions : il s'agit de la création autrichienne de Jenůfa (Ihre Ziehtochter) le 16 février 1918 au Hofoper - son auteur, Leoš Janáček, est certes originaire de Brno, mais il a acquis la célébrité à Prague et son œuvre occupe une position clef dans la vie musicale de la capitale tchèque ${ }^{27}$. Dans le contexte de la première guerre mondiale, l'œuvre a été perçue diversement : volonté d'apaisement des tensions politiques pour les uns, provocation inutile ou encore geste de réappropriation par l'Autriche d'une partie de sa culture au moment où celle-ci menace de lui échapper pour les autres ${ }^{28}$. Si, globalement, le ton de la presse viennoise est plutôt positif, on sent que les arrière-plans politiques et idéologiques sont présents dans toutes les consciences.

Ce sont souvent des considérations d'ordre idéologique qui font obstacle à la représentation d'une partie du répertoire opératique

25. Jan Löwenbach, "Tschechoslowakische Komponisten...», art. cité, p. 275-276 : "Steigerung und Komplizierung der technischen Ausdrucksmittel, [...] Auflösung der harmonischen Grundlagen, [...] Akzentuierung des rein Artistischen und Subjektiven [...] die tschechische Musik ringt nach neuem Ausdruck, der die gute Tradition zwar festhält, aber den Geist der neuen Zeit zu erfassen dürstet.»

26. Voir Max Brod, «Erinnerung an Janáček», Musikblätter des Anbruch, août-septembre 1928, p. 234 : «Die deutsche Uraufführung der Jenůfa in Wien (während des Krieges) war einige Jahre vor dem Berliner Sieg ohne besonders tiefe Wirkung geblieben.» Voir, dans ce numéro, l'article de Gaëlle Vassogne.

27. Voir notamment Jan Löwenbach, «Prager Spielzeit», Musikblätter des Anbruch, mai 1925, p. 284, ainsi que : Max Brod, «Erinnerung an Leoš Janáček», art. cité, p. 234.

28. Voir cette étonnante remarque sur Jenůfa dans Der Merker, vol. $9, \mathrm{n}^{\circ} 1$, janvier-mars 1918, p. 24 : «Doch daß österreichische Kunst im eigenen Land endlich auch zu Ehren gelangt, sei mit ehrlicher Freude herzlich begrüßt!» 
tchèque dans l'ancienne capitale des Habsbourg pendant toute la période de l'entre-deux-guerres. Vienne se vante certes d'être la ville qui a permis à Smetana d'accéder à un véritable rayonnement international $^{29}$, mais reste réticente envers l'opéra phare de la culture musicale tchèque, Libuše. Hanslick voyait déjà dans cet opéra un signe du déclin de Smetana ${ }^{30}$, gagné selon lui par le wagnérisme, et Mahler n'avait pas pu le faire jouer au K. k. Hof-Operntheater en raison de «réticences dynastiques ${ }^{31} »$. Il faut attendre 1924 et une tournée de la troupe d'Olomouc pour que l'œuvre soit donnée intégralement non pas à l'Opéra, mais au Metropoltheater, ce qui est l'occasion pour la presse viennoise de rappeler que cette "vision fantastique de l'existence éternelle du peuple de Bohême» qui sous-tend l'œuvre de Smetana devait nécessairement devenir «une pomme de discorde» pour les Autrichiens ${ }^{32}$.

Si la presse autrichienne en général et les Musikblätter des Anbruch en particulier parlent fréquemment de «musique tchèque» (mais très rarement de "musique bohème» ou de «musique morave», et jamais de «musique pragoise»), toute la vie musicale du pays semble se résumer à Prague - les villes de Brno ou Olomouc ne sont quasiment jamais évoquées, en dépit de leur importance artistique. La capitale de la Tchécoslovaquie apparaît comme le lieu où se cristallise toute la créativité des artistes tchèques, elle se nourrit aussi de tout ce que produit le monde rural bohème ou morave : la création musicale n'est pas une pure construction d'intellectuels urbains, mais puise dans les paysages et les traditions tchèques. En témoignent l'œuvre et la carrière du compositeur tchèque Josef Bohuslav Foerster (1859-1951),

29. Voir notamment Paul Stefan, "Friedrich Smetana und seine Verkaufte Braut», RadioWien, $\mathrm{n}^{\circ}$ 51, 20-26 septembre 1926, p. 2257 : «Es ist sehr schön und uns Wienern erfreulich, daß dieser Durchbruch eines Meisters, eines für die gesamte Menschheit bedeutungsvollen Geistes von Wien aus erfolgt ist»; ainsi que : Julius Korngold, «Friedrich Smetana. Zur Hundertsten Wiederkehr seines Geburtstags», art. cité, p. 2.

30. Eduard Hanslick, «Concerte» (Feuilleton), Neue Freie Presse, 4 mars 1890, p. 3.

31. Rudolf Stephan Hoffmann, «Die tschechische Oper in Wien», Musikblätter des Anbruch, juin-juillet 1924, p. 267 : «Man sah Kuß und Geheimnis, die seit Mahler verschwunden sind, Libussa, die er gegen dynastische Bedenken nicht hatte erzwingen können, und die ebenso unbekannten Zwei Witwen.»

32. Anonyme, "Theater- und Kunstnachrichten», Neue Freie Presse, 29 mai 1924, p. 10 : "Der Librettist [...] läßt Libussa in einer phantastischen Vision den ewigen Bestand des böhmischen Volkes prophezeien. Gerade diese Szene, die dem für die projektierte Prager Krönung Kaiser Franz Josefs 1871 komponierten Festspiel die höchste Weihe geben sollte, [...] mußte bei uns zum Stein des Anstoßes werden.» 
qui «bien que vivant à Prague», a grandi «au beau milieu de la nature et parmi les campagnards», trouvant l'inspiration dans la «fréquentation intense » de la campagne de Bohême tout en ayant accès, dans la capitale, "aux bienfaits d'une culture moderne et raffinée $e^{33}$ ». La ruralité reste donc, comme au $\mathrm{XIX}^{\mathrm{e}}$ siècle, l'univers poétique auquel puise la tradition nationale. La capitale tchèque offre certes aux artistes ses institutions et la possibilité d'accéder à la reconnaissance nationale, voire internationale, mais c'est dans les campagnes bohème et morave que les musiciens cherchent la substance poétique de leurs créations. On ne trouve d'ailleurs pas, dans les Musikblätter des Anbruch, d'article cherchant à montrer la ville de Prague comme un facteur poétogène dans la lignée de ce que dit Adorno lorsqu'il tente de justifier un parallèle entre Kafka et Janáček - et plus exactement entre le caractère fantastique de La Métamorphose (Die Verwandlung) et celui de L'Affaire Makropoulos (Věc Makropoulos) - en disant que l'écrivain et le compositeur vivaient tous les deux à Prague ${ }^{34}$.

La problématique d'une modernité qui parvient difficilement à se détacher de l'emprise du passé et de ses traditions apparait de manière emblématique dans l'introduction au numéro spécial consacré à la fête de la musique à Prague en 1925 rédigée par Paul Stefan : «La musique pragoise, c'est la tradition et l'histoire ${ }^{35} »$. Le rapport de la musique tchèque à la question nationale donne d'ailleurs lieu à des prises de position passablement contradictoires au sein de la revue viennoise. L'ancrage national de l'art des sons est affirmé avec force dans un article d'Erwin Felber, qui tout en étant un ardent défenseur de la nouvelle musique, n'hésite pas à reprendre des clichés éculés sur les caractéristiques musicales nationales et est profondément convaincu que la musique tchèque ne peut se développer qu'en puisant l'inspiration dans sa terre natale : «C'est l'esprit national de l'artiste, l'esprit propre

33. Zdeněk Nejedlý, «Tschechische Musik, III : Jos. B. Foerster», Musikblätter des Anbruch, février 1920, p. 100-101 : «Daneben aber unterhielt sein reger Verkehr mit dem Lande wieder lebhafte Vorstellungen in ihm von der Natur dort draußen [...] und so wuchs der junge Künstler, obschon er in Prag lebte, gleichsam mitten in der Natur und unter dem Landvolke auf, während ihm wieder Prag selbst die Möglichkeit bot, alle Gnaden der neuesten und verfeinertsten Kultur einzudringen.»

34. Theodor W. Adorno, «Frankfurter Opern- und Konzertkritiken», dans Gesammelte Schriften, 19: Musikalische Schriften VI, Francfort, Suhrkamp, 1984, p. 151. Notons au passage que l'attachement de Janáček aux traditions musicales populaires lui vaut un certain mépris de la part d'Adorno, qui ne voit en lui qu'un folkloriste.

35. Paul Stefan, «Prag», Musikfest in Prag. Sonderheft des Anbruch, mai 1925, p. 231 : «Prager Musik ist Überlieferung und Geschichte.» 
à sa race par-delà les mélanges du sang, ses prédispositions morales liées à la structure particulière de son cerveau qui se créent un monde sonore adéquat et mettent le matériau au service d'une idée technique déterminée $^{36}$.» Il ne faut toutefois pas se méprendre sur les convictions idéologiques de Felber : elles témoignent certes de préjugés et de clichés persistants, mais ne sauraient être assimilées à des théories raciales dont Felber sera lui-même victime peu après l'Anschluss. Il publie par ailleurs dans le même numéro de la revue un article consacré à la musique juive qui relève d'une forme de sionisme musical et jette un tout autre éclairage sur sa conception des rapports entre musique et nation ${ }^{37}$.

À l'opposé, la revue publie de nombreuses contributions qui contestent vigoureusement cette idéologie d'une musique émanant de l'esprit d'un peuple ou d'une nation. C'est le cas par exemple de Paul Stefan dans l'article qu'il consacre à Jenůfa. L'opéra de Janáček marque selon lui le dépassement du point de vue national et rural dans une universalité qui n'est pas sans rappeler Fidelio de Beethoven: la fin de l'opéra est ainsi interprétée comme la «résolution du rude conflit paysan dans la sphère de l'humanité supérieure». Et Paul Stefan ajoute : «Peut-on suggérer que cette sphère est celle de la réunion d'idéaux slaves et allemands ${ }^{38}$ ?" Le même sujet est abordé sur un ton bien plus polémique par Paul Bekker, qui prend position de façon virulente contre toute interprétation nationale ou nationaliste de la création musicale. Dans un article conçu comme une réponse à la polémique engagée par Hans Pfitzner contre la nouvelle musique dans un texte antisémite et raciste intitulé Die neue Ästhetik der musikalischen Impotenz (1920), Bekker argumente en disant que la critique musicale relève de l'esthétique et non de la généalogie et que toute discussion autour du caractère national (mais aussi international) de

36. Erwin Felber, «Gedanken über Musik und Volkstum», Anbruch, février 1928, p. 56 : "Der nationale, der trotz aller Blutmischung sagen wir rassenmäßige Geist des Künstlers, seine im besonderen Bau des Gehirnes begründeten seelischen Anlagen sind es, welche sich die ihnen angemessene Tonwelt schaffen, und das Material in den Dienst einer ganz bestimmten technischen Idee stellen.»

37. Voir Annkatrin Dahm, Der Topos der Juden: Studien zur Geschichte des Antisemitismus im deutschsprachigen Musikschrifttum, Göttingen, Vandenhoeck \& Ruprecht, 2007, p. 272.

38. Paul Stefan, «Der Erfolg der Jenůfa», Musikblätter des Anbruch, décembre 1925, p. 546 : «Aberganz besonders wertvoll scheint mir auch [...] die Lösung des grellen Bauernkonfliktes in einer Sphäre höherer Humanität. Darf man andeuten, daß es die Sphäre ist, in der sich slawische und deutsche Ideale vereinigen?» 
la musique est purement stérile. La mise en avant du critère national conduit selon lui «à un comportement haineux et néfaste, prenant un caractère stigmatisant ${ }^{39}{ }^{\prime}$.

La revue ne rend presque jamais compte directement des événements politiques, sinon de manière allusive. Même la prise de pouvoir par Hitler est passée sous silence - on notera toutefois l'exception notable d'un article publié en 1931 par un responsable des éditions Universal, Hans Heinsheimer, où il est question de cette «terreur secrète» responsable d'un "abêtissement invisible de l'opinion publique ${ }^{40}$ ». L'assombrissement du paysage politique dans les années 1930 pousse toutefois certains auteurs à prendre parti et à réaffirmer les valeurs auxquelles ils sont attachés. Dans son pamphlet contre l'«autarcie musicale», Stuckenschmidt constate que si, dans l'immédiat après-guerre, l'idée de progrès culturel telle que la défendait la revue pouvait constituer un frêle rempart contre les préjugés nationalistes et conservateurs, le défaitisme culturel qui s'empare de l'Europe au début des années 1930 fait obstacle à tout développement artistique : "Avec cette notion de tradition, on commet de nos jours de telles idioties dans la vie culturelle allemande que pratiquement toute discussion sur le renouveau artistique se trouve exclue ${ }^{41}$.» Avec ces remarques désabusées, Stuckenschmidt semble prédire la disparition programmée de la revue, qui ne survivra pas à l'Anschluss ${ }^{42}$. D'ailleurs le critique quittera la revue peu après la publication de cet article.

L'évolution du contexte politique aurait logiquement dû, si la revue Anbruch était restée fidèle à ses grands principes, rendre plus criant l'écart entre cet attachement des musicographes tchèques à la tradition

39. Paul Bekker, "Nationale und internationale Musik», art. cité, p. 176-177 : "Seine Behandlung führt nicht nur zu jener üblen, denunziatorisch zugespitzten Gehässigkeit, die heute eine der Hauptwaffen der Kritik geworden ist, sie schafft nicht nur jene Verwirrung der Begriffe und Anschauungen, wie sie sich in der Zerspaltung der Meinungen kundgibt, sie umnebelt vor allem den Blick und verwehrt ihm die wahre Erkenntnis der geistigen Zeitströmungen, die in jedem wahren Künstler, mag er sich national oder international gebärden, zutiefst wirken und das Schaffen bestimmen.»

40. Hans Heinsheimer, «Neues vom Tage», Anbruch, janvier 1931, p. 1 : «Und hier setzt der geheime Terror ein, die unsichtbare Verdummung der Öffentlichkeit.»

41. Hans Heinz Stuckenschmidt, «Musikalische Autarkie», art. cit., p. 105 : «Mit diesem Begriff der Tradition wird heutzutage im deutschen Kulturleben ein Unfug getrieben, der nahezu alle Diskussion über künstlerische Erneuerung ausschließt».

42. Le dernier numéro paraît en novembre 1937. Le numéro suivant, annoncé pour la mi-janvier 1938, ne paraîtra jamais et Paul Stefan, qui fut le dernier rédacteur en chef de la revue, devra bientôt prendre le chemin de l'exil. 
nationale et la volonté des contributeurs autrichiens et allemands de rompre, au nom de la modernité, avec cette tradition et avec le nationalisme qui en fait l'apologie. Or, il n'en est rien : Anbruch, qui perd successivement ses contributeurs les plus marquants (Bekker, Adorno, Stuckenschmidt), va peu à peu se plier subrepticement à l'idéologie dominante, se désintéresser de la création contemporaine, qu'elle soit tchèque ou allemande, et perdre son caractère international en se concentrant de plus en plus ostensiblement sur la vie musicale autrichienne. En 1935, elle adopte un nouveau titre : Anbruch. Österreichische Zeitschrift für Musik. Ce n'est pas un hasard si le nombre d'articles consacrés à Bruckner, l'un des compositeurs de prédilection de la propagande imaginée par Goebbels, explose à partir de cette date qui marque le début du déclin définitif d'une revue qui fut auparavant une tribune ouverte à tous les représentants de la modernité. 